\title{
Sample Entropy-Based Approach to Evaluate the Stability of Double-Wire Pulsed MIG Welding
}

\author{
Ping Yao, ${ }^{1}$ JiaXiang Xue, ${ }^{2}$ Kang Zhou, ${ }^{3}$ and XiaoJun Wang ${ }^{1}$ \\ ${ }^{1}$ College of Electromechanical Engineering, Guangdong Polytechnic Normal University, Guangzhou 510635, China \\ ${ }^{2}$ School of Mechanical and Automotive Engineering, South China University of Technology, Guangzhou 510640, China \\ ${ }^{3}$ Department of Mechanical and Aerospace Engineering, Hong Kong University of Science and Technology, Hong Kong
}

Correspondence should be addressed to Ping Yao; ypsunny@163.com

Received 15 March 2014; Revised 9 May 2014; Accepted 9 May 2014; Published 3 June 2014

Academic Editor: Hamid Reza Karimi

Copyright (C) 2014 Ping Yao et al. This is an open access article distributed under the Creative Commons Attribution License, which permits unrestricted use, distribution, and reproduction in any medium, provided the original work is properly cited.

\begin{abstract}
According to the sample entropy, this paper deals with a quantitative method to evaluate the current stability in double-wire pulsed MIG welding. Firstly, the sample entropy of current signals with different stability but the same parameters is calculated. The results show that the more stable the current, the smaller the value and the standard deviation of sample entropy. Secondly, four parameters, which are pulse width, peak current, base current, and frequency, are selected for four-level three-factor orthogonal experiment. The calculation and analysis of desired signals indicate that sample entropy values are affected by welding current parameters. Then, a quantitative method based on sample entropy is proposed. The experiment results show that the method can preferably quantify the welding current stability.
\end{abstract}

\section{Introduction}

Double-wire pulsed MIG welding is used prevalently in current industrial occasions, such as engineering machinery, coal mining, and ship building. For achieving better operation efficiency and gaining satisfactory welding operation, appropriate process control methods need to be used [1-3]. Electric signals contain a lot of information reflecting the welding performance and welding quality; online measuring of the corresponding electric signals can do a lot of useful things, such as power factor correction $[4,5]$ and fault diagnosis $[6,7]$. Analyzing and processing this information can help us to improve welding stability and to objectively assess welding performance.

For decades, a lot of relative researches were taken to use the electrical signals to analyze the welding process and improve the production. Luksa and Rymarski evaluated welding quality by analyzing the changes of electric signals [8]. Shinoda et al. found that splashes result in unstable electric signals and affect welding quality [9]. Hermans and den Ouden found that welding process stability is directly relative to weld pool oscillation [10]. Quinn et al. evaluated welding quality by setting stable current thresholds [11]. Adolfsson et al. studied a repetition sequential probability ratio algorithm. This algorithm can detect small sudden changes in electric signals [12]. Klimov et al. designed a new method of collecting current signals in the welding. By means of the method, current signals of ERW were collected for welding quality evaluation [13]. Šoštarić et al. indicated that online monitoring of the welding process by electric signals is feasible after comparing the quality of online monitoring signals and oscilloscope-acquired signals [14]. $\mathrm{He}$ et al. assessed the stability of submerged arc welding by analyzing current signals with Lyapunov exponent [15]. Xue et al. analyzed the effects of current and voltage on welding stability from the perspective of statistics and spectrum $[16$, 17]. Li et al. and Chen et al. established a multi-information acquisition system to collect a variety of electrical signals for analyzing the welding process $[18,19]$. Chen et al. considered that relatively stable welding process can be obtained only under optimum operations conditions by analyzing the effect of current waveform on the stability of short circuiting transfer process [20]. 
Hence, using electrical signals in welding production process is very usual in reality. However, present studies of welding current signals mainly focused on time domain and frequency domain. In practical production occasion, current signals change in chaos. In other words, the actual process is typically nonstationary and random. Hence, in addition to the features of time domain and frequency domain, nonlinear features of welding current should also be concerned further by researchers.

There were some relative researches focused on the issue of nonlinear features of the welding current. Entropy is a common function which is used to describe the system state. It is used to calculate system disorder phenomena. In recent years, researchers have made some studies about welding process stability by means of approximate entropy (ApEn). ApEn was proposed by Pincus for overcoming the difficulty in solving the entropy in chaos [21-24]. Pincus and Kalman found that the ApEn of arc voltage increases with the increase of wire feed speed in GMAW [24]. Cao et al. analyzed the relationship between ApEn (and its standard) and the stability of short circuit transfer of $\mathrm{CO}_{2}$ welding $[25,26]$. Nie et al. used neural network to predict the ApEn of P-MIG welding of aluminum alloy and achieved good results [27]. Zhang et al. evaluated adaptive control results by ApEn and found that smaller ApEn leads to better adaptive control [28].

The study showed that there is a certain relationship between the entropy and stability of welding signals, but the consistency is relatively poor due to being affected by various data length and embedding dimension. In 2000, Richman and Moorman proposed a new time series complexity measurement method on the basis of ApEn-sample entropy (SampEn) [29]. Moreover, SampEn reduces ApEn errors, and better consistency and accuracy can be gained with faster computing speed [30].

Current researches of the SampEn focus on the biomedical field [31-33] and gain better achievements in the analysis of atrial fibrillation electrocardiograms [34]. An intelligent prognostic for battery health based on SampEn feature of discharge voltage was also proposed [35].

The study showed that the usage of SampEn electrical stability analysis is feasible. Experimental results show that the SampEn method can accurately quantify the stability of the welding process. Hence, it has great potential in research and quality analysis of welding mechanism.

The rest of this paper is organized as follows. In Section 2, the flow of Richman's algorithm is stated. The relation between sample entropy and stability of welding signals is explored in Section 3. Also, in Section 4, the effect of different current variables on the other variables on the SampEn is illustrated in detail. To validate the proposed method, a new evaluating current stability is given in Section 5 , and then the actual experiments and corresponding analysis are provided subsequently.

\section{Algorithm}

In this work, Richman's algorithm is used. First of all, algorithm process is introduced as follows.
Given a sequence number with $N$ points $u(1), u(2) \cdots$ $u(N)$ as original data. When embedding dimension is $m$ and $m+1$, before $N-m$, vectors need to satisfy the following condition: $m$-dimensional vector $X_{m}(i)$ is meaningful when $1 \leq i \leq N-m$.

(1) Form vector sequences of size $m$ :

$$
\begin{array}{r}
X_{m}(i)=[u(i), u(i+1), u(i+m-1)], \\
i=1,2, \ldots, N-m+1 .
\end{array}
$$

(2) Define the distance between $X_{m}(i)$ and $X_{m}(j)\left(d\left[X_{m}(i), X_{m}(j)\right]\right)$ as the absolute maximum difference between their scalar components:

$d\left[X_{m}(i), X_{m}(j)\right]=\max _{k=0 \cdots m-1}(|u(i+k)-u(j+k)|)$.

(3) For a given $X_{m}(i)$, count the number of $j(1 \leq j \leq$ $N-m, j \neq i)$, denoted as $N^{m}(i)$, such that the distance between $X_{m}(i)$ and $X_{m}(j)$ is less than or equal to $r$. Then, for $1 \leq i \leq N-m$,

$$
B_{r}^{m}(i)=\frac{N^{m}(i)}{N-m} .
$$

(4) Define $B_{r}^{m}(i)$ as

$$
B^{m}(r)=\frac{\sum_{i=1}^{N-m+1} B_{i}^{m}(r)}{N-m+1} .
$$

(5) Increase the dimension to $m+1$ :

$$
\begin{array}{r}
X_{m+1}(i)=[u(i), u(i+1), u(i+m)], \\
i=1,2, \ldots, N-m .
\end{array}
$$

(6) Calculate $N^{m}(i)$ as the number of $X_{m+1}(i)$ within $r$ of $X_{m+1}(j)$, where $j$ ranges from 1 to $N-m(j \neq i)$. Then, $B_{r}^{m+1}(r)$ is defined as

$$
B_{r}^{m+1}(r)=\frac{N^{m}(i)}{N-m-1} .
$$

(7) Set $B^{m+1}(r)$ as

$$
B^{m+1}(r)=\frac{\sum_{i=1}^{N-m} B_{i}^{m+1}(r)}{N-m} .
$$

(8) Finally, SampEn can be defined as

$$
\operatorname{SampEn}(m, r, N)=\lim \left\{-\operatorname{In}\left[\frac{B^{m+1}}{B^{m}(r)}\right]\right\},
$$

which is estimated by the following statistic:

$$
\operatorname{SampEn}(m, r, N)=-\operatorname{In}\left[\frac{B^{m+1}}{B^{m}(r)}\right] .
$$




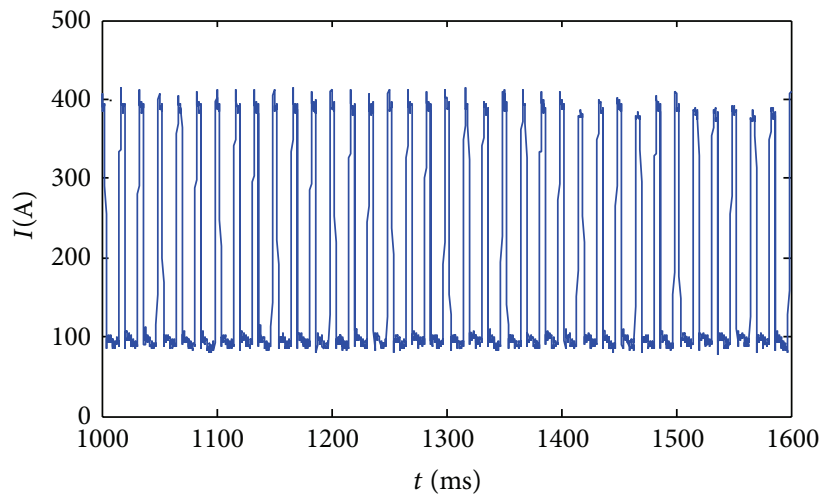

(a)

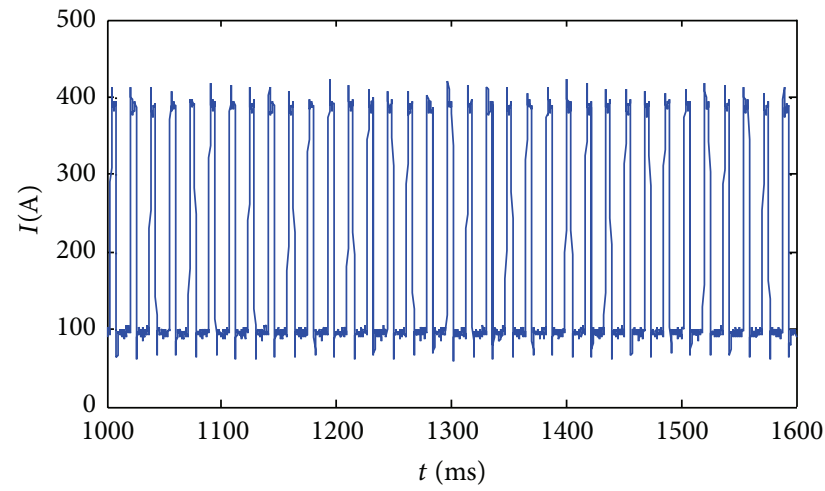

(c)

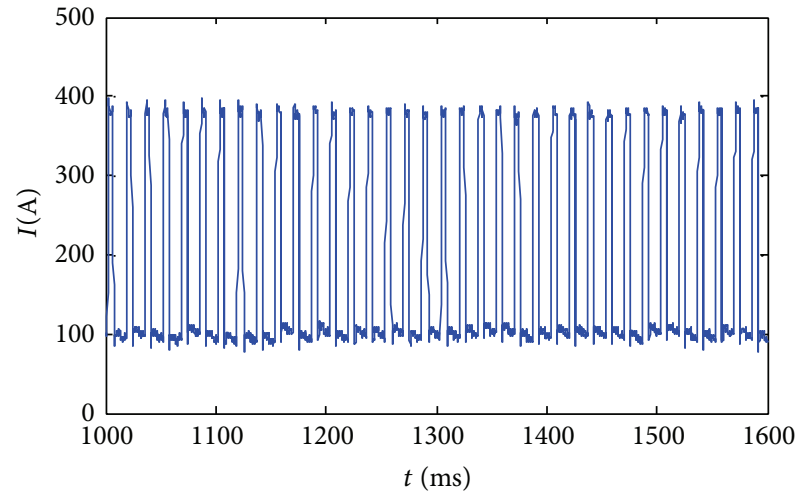

(b)

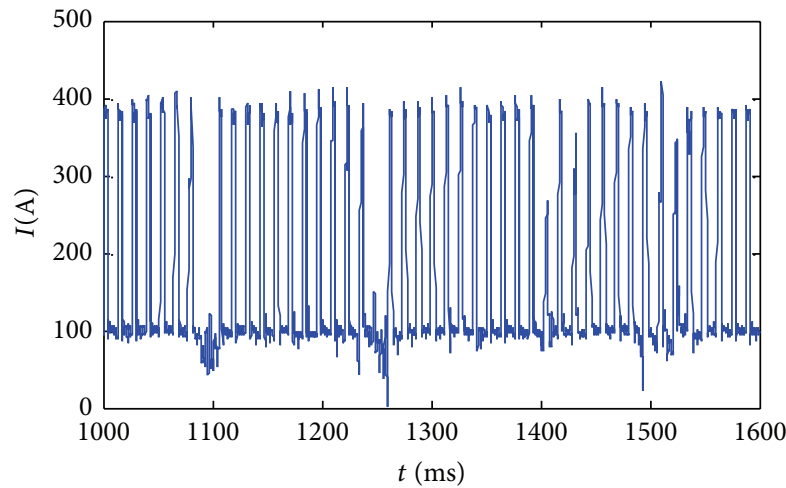

(d)

FIGURE 1: Current waveform: (a) signal 1, (b) signal 2, (c) signal 3, and (d) signal 4.

From step (1) to step (7), ir shows that SampEn calculation requires determining three parameters, which are $m$, $r$, and $N$, where different $m$ and $r$ correspond to different SampEn. Presently, there are no theories guiding how to assign values to $m$ and $r$. In the current study, the usual assigned value of $m$ was 1 or 2 , and the value of $r$ was 0.1 to 0.5 times of the standard deviation of raw data. Lake et al. thought that the SampEn distribution is normal when $m$ is smaller and $r$ is larger, and small amount of lost data points does not affect the entropy calculation [36].

Consequently, in this paper, the SampEn is calculated under the following conditions:

$$
m=2, \quad r=0.07, \quad N=2000 .
$$

\section{SampEn and Stability}

To explore the relation between sample entropy and stability of welding signals, four current signals which have the same parameters but different stability are chosen to do the calculation of SampEn and its corresponding standard deviations (SD). Four signals of different stability are selected. Their parameters are as follows: $33.3 \%$ of pulse width (\% of period, PW), $380 \mathrm{~A}$ of peak current $\left(I_{p}\right)$, and $100 \mathrm{~A}$ of base current $\left(I_{b}\right)$ and of frequency $(f)$. Wavelet analyzer is used to capture current signals; its presentation is shown in Figure 1. From signal 1 to signal 4, the stability decreases in turn.
SampEn calculation results are shown in Figure 2. SampEn values of signal 1 and signal 2 are relatively small and have little difference, while SampEn value of signal 3 is more stable and larger than the preceding two signals. The magnitude changes of signal 4 are much larger than the preceding three signals.

Figure 3 shows the SampEn averages (AVG) and SD of 20 calculations; SD reflects the degree of deviation from the average of SampEn sequence. As shown in Figure 3, SD of signal 4 is about twice those of the preceding three signals, which shows that the stability of signal 4 is diverse at different time. Comparing Figures 1, 2, and 3, it is found that the more stable the signal, the smaller the AVG and SD. This is because the randomness, irregularities, and SampEn on the time series decrease.

\section{Parameters Effects}

Double-wire pulsed MIG welding has more different parameters when compared to other types of welding operation. For different electrical signals which serve different welding occasions, it is insufficient to only compare their SampEn values; the effect of different current variables on the SampEn is required to be focused on.

In order to analyze the parameters' effects on evaluation results, the orthogonal experiment was carried out in this 


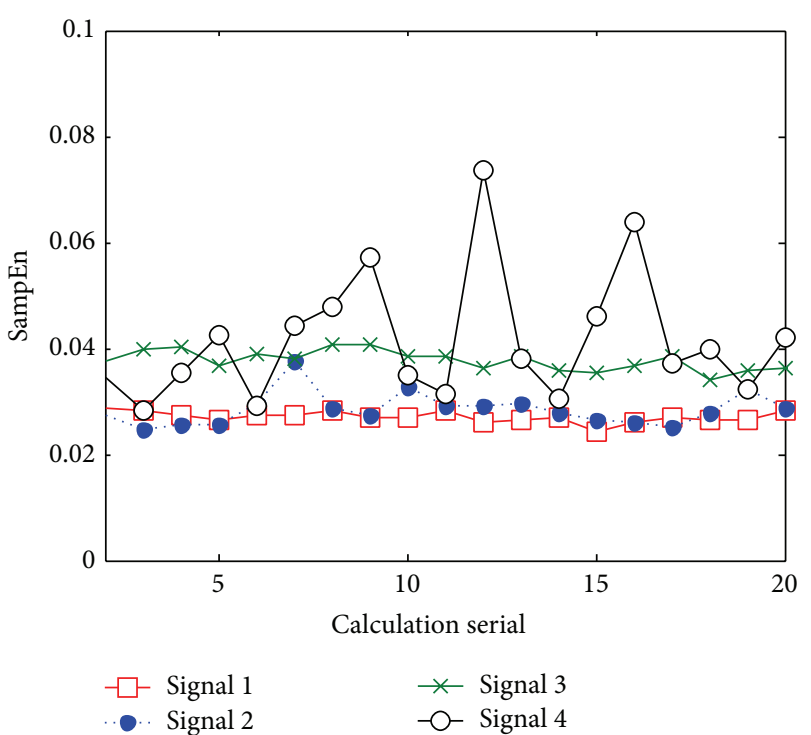

FIgURE 2: Calculation results of SampEn.

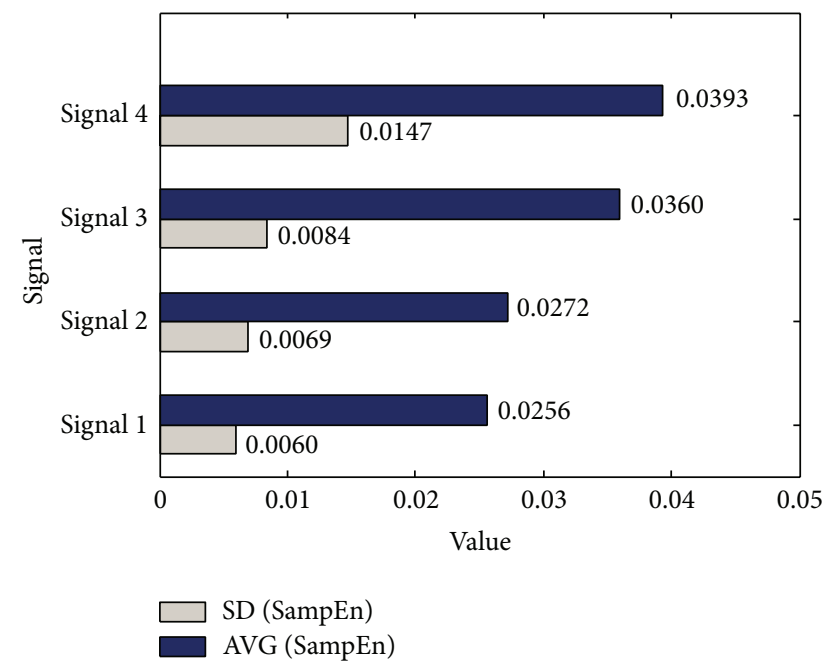

FIgURE 3: AVG and SD of SampEn.

work, and we choose four factors, which are PW, $I_{p}, I_{b}$, and $f$, and these four factors are chosen as research subjects. For each factor, three levels are selected from small to large. L9 $\left(3^{4}\right)$ orthogonal table is used. Using Matlab Simulink establishes a simulation model to generate 9 desired signals. Then, SampEn values of the 9 desired signals are calculated. Corresponding experimental arrangement is shown in Table 1.

Table 1 shows that the variation of the current parameters has certain impacts on SampEn values. In Table 1, maximum SampEn value is 0.0305 , while minimum SampEn value is only 0.0074 , which means that there is large difference between them. Range analysis results are shown in Table 2.

The frequency has the largest impacts on results among these four factors, and its corresponding range value is 0.0179 .
TABLE 1: Orthogonal arrangement and results.

\begin{tabular}{lccccc}
\hline Number & PW/\% & $I_{p} / \mathrm{A}$ & $I_{b} / \mathrm{A}$ & $f / \mathrm{Hz}$ & $\mathrm{AVG}$ \\
\hline 1 & 50 & 450 & 100 & 150 & 0.0305 \\
2 & 50 & 300 & 80 & 100 & 0.0202 \\
3 & 50 & 200 & 50 & 50 & 0.0101 \\
4 & 33 & 450 & 80 & 50 & 0.0090 \\
5 & 33 & 300 & 50 & 150 & 0.0275 \\
6 & 33 & 200 & 100 & 100 & 0.0181 \\
7 & 20 & 450 & 50 & 100 & 0.0147 \\
8 & 20 & 300 & 100 & 50 & 0.0074 \\
9 & 20 & 200 & 80 & 150 & 0.0222 \\
\hline
\end{tabular}

TABLE 2: Range analysis of AVG.

\begin{tabular}{lcccc}
\hline SampEn & PW & $I_{p}$ & $I_{b}$ & $f$ \\
\hline $\mathrm{AVG}_{1}$ & 0.0608 & 0.0542 & 0.056 & 0.0802 \\
$\mathrm{AVG}_{2}$ & 0.0546 & 0.0551 & 0.0514 & 0.053 \\
$\mathrm{AVG}_{3}$ & 0.0443 & 0.0504 & 0.0523 & 0.0265 \\
Min & 0.0148 & 0.0168 & 0.0171 & 0.0088 \\
Max & 0.0203 & 0.0184 & 0.0187 & 0.0267 \\
Range & 0.0055 & 0.0016 & 0.0015 & 0.0179 \\
\hline
\end{tabular}

With the change in $f$ levels, the AVG rapidly reduces from 0.0802 to 0.0265 ; followed by the PW, the corresponding range value is $0.0055 ; I_{p}$ and $I_{b}$ have little effect on the results, and their corresponding range values are 0.0016 and 0.0015 , respectively. In Table 1, the desired signals have the same stability; however, their SampEn values are different. Hence, the effects of current parameters on SampEn values should be considered when SampEn is used for quantifying current signals stability.

SampEn values change with parameters because varying patterns of time series are different under different parameters. For example, when frequency is higher, time series change more quickly; so the probability of appearance of a new model increases in the same data segment, and its corresponding SampEn values are also bigger.

\section{Evaluation Method}

According to the researches described in Sections 3 and 4, not only the electrical signals but also the parameters' values can affect the SampEn. Hence, to further objectively evaluate the electrical signals and exclude the influence of different parameters on SampEn, a new method, which is used to evaluate the current stability of double-wire pulsed MIG welding, is designed in this paper.

\subsection{Method}

(1) The SampEn stability evaluation index of single current (CSI) is calculated as follows:

$$
\mathrm{CSI}=\frac{\mathrm{AVG}-\mathrm{AVG}_{I}}{\mathrm{AVG}_{I}} \times \frac{\mathrm{SD}-\mathrm{SD}_{I}}{\mathrm{SD}_{I}},
$$




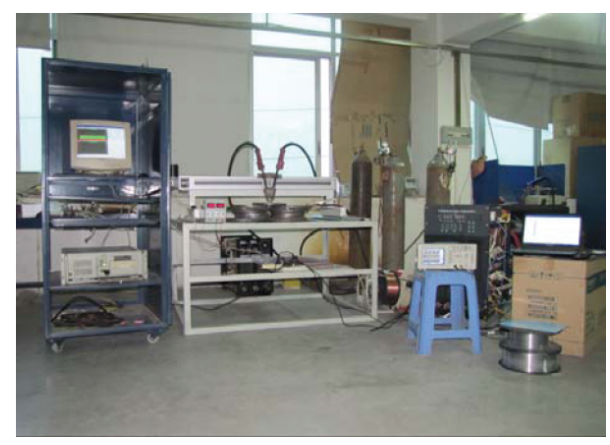

Figure 4: The platform of experiment.

where AVG and SD represent averages and standard deviations of original signals SampEn, while $\mathrm{AVG}_{I}$ and $\mathrm{SD}_{I}$ represent averages and standard deviations of desired signals SampEn.

(2) Calculate subindex of double-wire $\left(\mathrm{DI}_{1}\right)$ :

$$
\mathrm{DI}_{1}=\alpha \times \max \left(\mathrm{CSI}_{1}, \mathrm{CSI}_{2}\right),
$$

where $\alpha$ represents weighting factor, which is generally determined based on wire and other conditions, while $\mathrm{CSI}_{1}$ and $\mathrm{CSI}_{2}$ represent stability evaluation indexes of leading current and trailing current, respectively.

(3) Calculate subindex of double-wire $\left(\mathrm{DI}_{2}\right)$ :

$$
\mathrm{DI}_{2}=(1-\alpha) \times \min \left(\mathrm{CSI}_{1}, \mathrm{CSI}_{2}\right) \text {. }
$$

(4) Calculate SampEn evaluation index of double-wire (DCSI):

$$
\mathrm{DCSI}=\mathrm{DI}_{1}+\mathrm{DI}_{2}
$$

5.2. Experiment. For validating the above method, corresponding experiments were conducted. A self-developed inverter welding machine is used for pulsed MIG welding. Q235 steel $(\delta=4.0 \mathrm{~mm})$ is used as bead on plate welds. Welding wire is $\mathrm{H} 08 \mathrm{Mn} 2 \mathrm{Si}(\Phi=1.0 \mathrm{~mm})$. The shielding gas is pure argon, and the flow rate is $15 \mathrm{~L} / \mathrm{min}$. Figure 4 shows the experimental platform.

Three double-wire current signals of different parameters and stability are selected for studying. Figure 5 shows the realtime current waveforms of stable signal to unstable signal, where ILeading is the abbreviation of leader wire current, while ITrailing is the abbreviation of trailing wire current. These corresponding parameters are in Table 3.

The current waveforms of ILeading1 and ITrailing1 in Figure 5(a) are regular and stable. On the contrary, the current waveforms in Figure 5(b) are relatively bad; especially greater spikes appear in the rise and fall in ILeading2, which means that there are greater overshoots, while ITrailing2 is relatively good. The stability of current waveforms in Figure 5(c) deteriorates; in ILeading3, large breaking arc
TABLE 3: Signal parameters.

\begin{tabular}{lcccc}
\hline Signals & $\mathrm{PW} / \%$ & $I_{p} / \mathrm{A}$ & $I_{b} / \mathrm{A}$ & $f / \mathrm{Hz}$ \\
\hline ILeading1 & 28.57 & 310 & 66 & 47.6 \\
ITrailing1 & 28.57 & 270 & 70 & 47.6 \\
ILeading2 & 39.13 & 305 & 70 & 86.9 \\
ITrailing2 & 39.13 & 252 & 68 & 86.9 \\
ILeading3 & 31.25 & 380 & 100 & 62.5 \\
ITrailing3 & 31.25 & 310 & 90 & 62.5 \\
\hline
\end{tabular}

appears at $2000 \mathrm{~ms}$, spikes can be seen in the base time from $2020 \mathrm{~ms}$ to $2110 \mathrm{~ms}$, the peak current begins to increase when the value achieves the $2120 \mathrm{~ms}$; the stability of ITrailing 3 is even worse, because the current is unable to reach a given value.

5.3. Analysis. According to the method described in Section 5.1, the value of $\alpha$ in (11), (12) is assumed as 0.75 , and the results are shown in Table 4 . As it can be found, CSI changes with the variation of current. The current waveforms of ILeading1 and ITrailing1 are regular and stable, which is corresponding to small CSI. The stability of ILeading2 is not as good as ILeading1; hence, its corresponding CSI is larger. ITrailing2 is relatively stable; therefore, its corresponding CSI is close to ITrailing1. The stability of ILeading3 deteriorates, and the corresponding CSI further increases. Moreover, ITrailing3 is very unstable; its CSI is also much larger than the others. Experiments show that CSI can truthfully reflect the stability of welding electrical signals.

DCSI of stable signal is only 1.5408 and DCSI of signal 2 is about twice that of stable signal, which indicates that the stability of signal 2 deteriorates. DCSI of signal 3 is 8.25 times the DCSI of signal 1, which indicates that signal 3 is very unstable. By this method, the stability of double-wire welding current is effectively distinguished. However, the present study sample size is so limited; hence, it is necessary to further study the robustness and the selection rule of weighting coefficients $\alpha$.

\section{Conclusions}

The relationship between SampEn and the current stability is studied. The results show that the more stable the current, the smaller the value and the standard deviation of SampEn under the same parameters. When welding current parameters are different, SampEn values are also different. Therefore, the welding current parameters' impacts should be considered when evaluating the welding stability. Then, a method of quantifying the stability of double-wire welding process based on SampEn is designed. The method puts forward a new idea for the quantitative evaluation of the welding process. The research is important for in-depth study of the law of the welding process and its stability mechanism. 


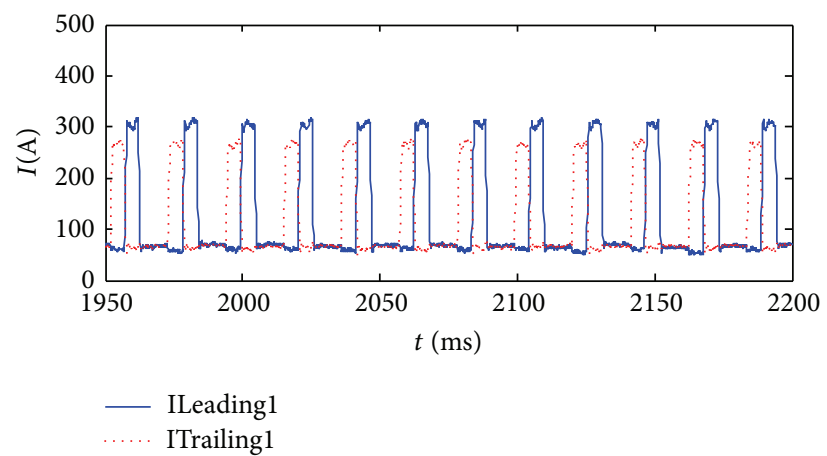

(a)

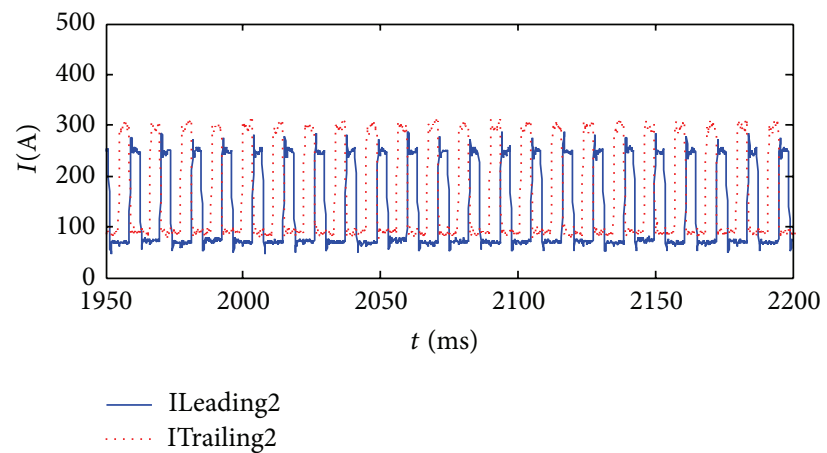

(b)

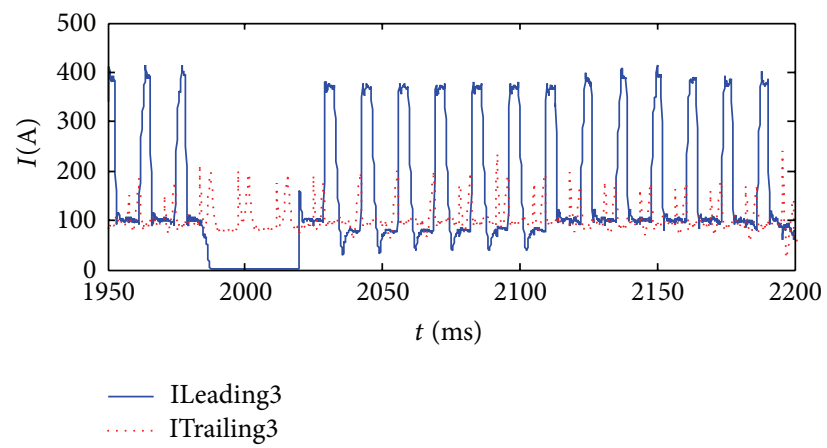

(c)

Figure 5: Double-wire current signals: (a) stable, (b) relatively stable, and (c) unstable.

TABLE 4: Signal parameters and quantitative results.

\begin{tabular}{lccccccc}
\hline Signal & $\mathrm{AVG}$ & $\mathrm{AVG}_{I}$ & $\mathrm{SD}$ & $\mathrm{SD}_{I}$ & $\mathrm{CSI}$ & $\mathrm{DI}_{1}$ & $\mathrm{DI}_{2}$ \\
\hline ILeading1 & 0.0198 & 0.0072 & 0.0049 & 0.0026 & 1.5206 & - & 0.3802 \\
ITrailing1 & 0.0204 & 0.0072 & 0.0048 & 0.0026 & 1.5476 & 1.1607 & - \\
ILeading2 & 0.0539 & 0.0151 & 0.0124 & 0.0053 & 3.4534 & 2.5900 & - \\
ITrailing2 & 0.0432 & 0.0151 & 0.0100 & 0.0053 & 1.6421 & - & 0.4105 \\
ILeading3 & 0.0340 & 0.0099 & 0.0116 & 0.0035 & 5.6308 & - & 1.4077 \\
ITrailing3 & 0.0570 & 0.0099 & 0.0146 & 0.0035 & 15.0685 & 11.3014 & - \\
\hline
\end{tabular}




\section{Conflict of Interests}

The authors declare that there is no conflict of interests regarding the publication of this paper.

\section{Acknowledgment}

The authors would like to thank the Foundation for Distinguished Young Teachers' Training of Guangdong (Grant no. Yq2013106).

\section{References}

[1] H. Y. Li, J. Y. Yu, C. Hilton, and H. H. Liu, "Adaptive slidingmode control for nonlinear active suspension vehicle systems using T-S fuzzy approach," IEEE Transactions on Industrial Electronics, vol. 60, no. 8, pp. 3328-3338, 2013.

[2] K. Zhou and L. L. Cai, "A nonlinear current control method for resistance spot welding," IEEE/ASME Transactions on Mechatronics, vol. 19, no. 2, pp. 559-569, 2013.

[3] X. Zhang, X. Lin, and Y. T. Wang, "Robust fault detection filter design for a class of neutral-type neural networks with timevarying discrete and unbounded distributed delays," Optimal Control Applications and Methods, vol. 34, no. 5, pp. 590-607, 2013.

[4] K. Zhou and L. L. Cai, "Online measuring power factor in AC resistance spot welding," IEEE Transactions on Industrial Electronics, vol. 61, no. 1, pp. 575-582, 2014.

[5] S. Yin, S. X. Ding, A. Haghani, H. Hao, and P. Zhang, "A comparison study of basic data-driven fault diagnosis and process monitoring methods on the benchmark Tennessee Eastman process," Journal of Process Control, vol. 22, no. 9, pp. 1567-1581, 2012.

[6] H. R. Karimi, "Robust synchronization and fault detection of uncertain master-slave systems with mixed time-varying delays and nonlinear perturbations," International Journal of Control, Automation and Systems, vol. 9, no. 4, pp. 671-680, 2011.

[7] S. Yin, X. B. Yang, and H. R. Karimi, "Data-driven adaptive observer for fault diagnosis," Mathematical Problems in Engineering, vol. 2012, Article ID 832836, 21 pages, 2012.

[8] K. Luksa and Z. Rymarski, "Collection of arc welding process data," Journal of Achievements in Materials and Manufacturing Engineering, vol. 17, pp. 377-380, 2006.

[9] T. Shinoda, H. Kaneda, and Y. Takeuchi, "An evaluation of short circuiting arc phenomena in GMA welding," Welding and Metal Fabrication, vol. 57, no. 10, pp. 522-525, 1989.

[10] M. Hermans and G. den Ouden, "Process behavior and stability in short circuit gas metal arc welding," Welding Journal, vol. 78, no. 4, pp. 137-141, 1999.

[11] T. P. Quinn, C. Smith, C. N. McCowan, E. Blachowiak, and R. B. Madigan, "Arc sensing for defects in constant-voltage gas metal arc welding," Welding Journal, vol. 78, no. 9, pp. 322-328, 1999.

[12] S. Adolfsson, A. Bahrami, G. Bolmsjö, and I. Claesson, "Online quality monitoring in short-circuit gas metal arc welding," Welding Journal, vol. 78, no. 2, pp. 59-73, 1999.

[13] A. S. Klimov, A. K. Kudinov, A. V. Komirenko, and A. N. Antsiborov, "A method for measuring current in resistance welding," Welding International, vol. 27, no. 10, pp. 830-833, 2013.
[14] D. Šoštaric, D. Vinko, D. Žagar, and I. Samardžic, "Reliability of welding parameters monitoring system," Strojarstvo, vol. 52, pp. 217-226, 2010.

[15] K. F. He, Q. Li, and J. Chen, "An arc stability evaluation approach for SW AC SAW based on Lyapunov exponent of welding current," Measurement, vol. 46, no. 1, pp. 272-278, 2013.

[16] J. X. Xue, Z. P. Yi, P. Fang, and L. Jia, "Study on virtual instrument for analyzing electrical signal of welding process," Chinese Journal of Mechanical Engineering, vol. 40, no. 2, pp. 6063, 2004 (Chinese).

[17] P. Yao, Intelligent control strategies and performance evaluation of integrated double wire arc welding power source, [Ph. D. Dissertation], South China University of Technology, Guangzhou, China, 2012, (Chinese).

[18] H. Li, J. H. Wang, D. F. Ai, and Y. Gao, "Synchronous multiinformation acquisition system for arc welding process," Journal of Tianjin University, vol. 45, pp. 184-189, 2012 (Chinese).

[19] B. Chen, J. F. Wang, and S. B. Chen, "A study on application of multi-sensor information fusion in pulsed GTAW," Industrial Robot, vol. 37, no. 2, pp. 168-176, 2010.

[20] M. A. Chen, Y. N. Jiang, and C. S. Wu, "Effect of current waveform on metal transfer in controlled short circuiting gas metal arc welding," Advanced Materials Research, vol. 718-720, pp. 202-208, 2013.

[21] S. M. Pincus, "Approximate entropy as a measure of system complexity," Proceedings of the National Academy of Sciences of the United States of America, vol. 88, no. 6, pp. 2297-2301, 1991.

[22] S. M. Pincus, "Approximate entropy (ApEn) as a complexity measure," Chaos, vol. 5, no. 1, pp. 110-117, 1995.

[23] S. M. Pincus, I. M. Gladstone, and R. A. Ehrenkranz, "A regularity statistic for medical data analysis," Journal of Clinical Monitoring, vol. 7, no. 4, pp. 335-345, 1991.

[24] S. Pincus and R. E. Kalman, "Irregularity, volatility, risk, and financial market time series," Proceedings of the National Academy of Sciences of the United States of America, vol. 101, no. 38, pp. 13709-13714, 2004.

[25] B. Cao, Y. P. Xiang, X. Q. Lü, M. Zeng, and S. S. Huang, "Approximate entropy-a new statistic to quantify arc and welding process stability in short-circuiting gas metal arc welding," Chinese Physics B, vol. 17, no. 3, pp. 865-877, 2008.

[26] B. Cao, X. Q. Lü, M. Zeng, Z. M. Wang, and S. S. Huang, "Approximate entropy analysis of current in short-circuiting arc welding," Acta Physica Sinica, vol. 55, no. 4, pp. 1696-1705, 2006 (Chinese)

[27] J. Nie, Y. Shi, J. K. Huang, and D. Fang, "Approximate entropy GRNN forecast for aluminum alloy pulsed MIG welding stability," Transactions of the China Welding Institution, vol. 31, no. 8, pp. 77-80, 2010 (Chinese).

[28] W. C. Zhang, L. J. Yang, and X. Q. Lü, "Approximate entropy based meso-spray transfer analysis of Al-alloy pulsed metal inert-gas welding under self-adapting control," Acta Physica Sinica, vol. 60, no. 2, Article ID 020601, 2011 (Chinese).

[29] J. S. Richman and J. R. Moorman, "Physiological time-series analysis using approximate and sample entropy," American Journal of Physiology: Heart and Circulatory Physiology, vol. 278, no. 6, pp. 2039-2049, 2000.

[30] R. Alcaraz and J. J. Rieta, "A novel application of sample entropy to the electrocardiogram of atrial fibrillation," Nonlinear Analysis: Real World Applications, vol. 11, no. 2, pp. 1026-1035, 2010.

[31] H. M. Al-Angari and A. V. Sahakian, "Use of sample entropy approach to study heart rate variability in obstructive sleep 
apnea syndrome," IEEE Transactions on Biomedical Engineering, vol. 54, no. 10, pp. 1900-1904, 2007.

[32] N. Castro, J. Grimaldos, O. Escalona, V. Kodoth, E. Lau, and G. Manoharan, "Assessment of atrial fibrillation organization during internal electrical cardioversion using sample entropy," in Proceedings of the Andean Region International Conference (Andescon '12), pp. 191-194, Cuenca, Ecuador, November 2012.

[33] R. Menayo, A. Encarnación, G. M. Gea, and P. J. Marcos, "Sample entropy-based analysis of differential and traditional training effects on dynamic balance in healthy people," Journal of Motor Behavior, vol. 46, no. 2, pp. 73-82, 2014.

[34] R. Alcaraz and J. J. Rieta, "A review on sample entropy applications for the non-invasive analysis of atrial fibrillation electrocardiograms," Biomedical Signal Processing and Control, vol. 5, no. 1, pp. 1-14, 2010.

[35] A. Widodo, M. Shim, W. Caesarendra, and B. Yang, "Intelligent prognostics for battery health monitoring based on sample entropy," Expert Systems with Applications, vol. 38, no. 9, pp. 11763-11769, 2011.

[36] D. E. Lake, J. S. Richman, M. P. Griffin, and J. R. Moorman, "Sample entropy analysis of neonatal heart rate variability," American Journal of Physiology: Regulatory Integrative and Comparative Physiology, vol. 283, no. 3, pp. R789-R797, 2002. 


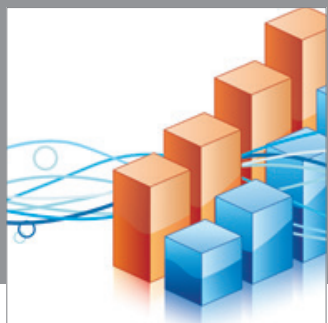

Advances in

Operations Research

mansans

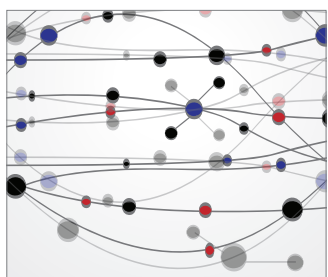

The Scientific World Journal
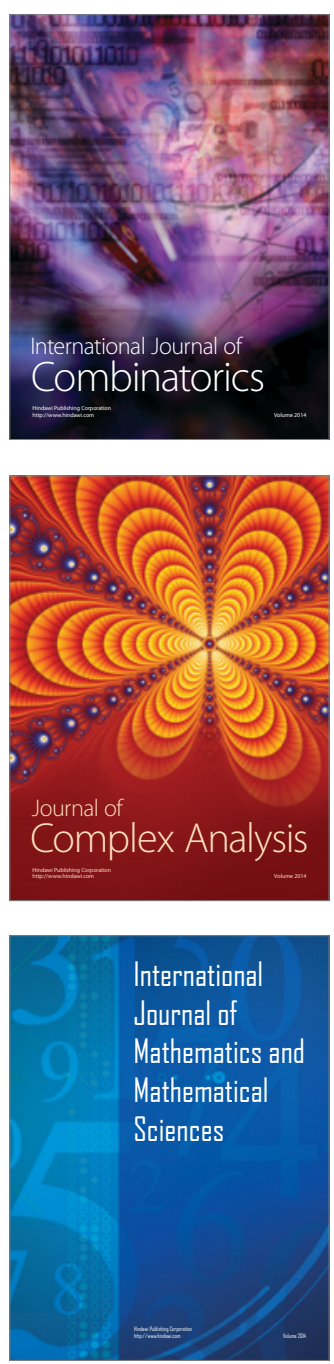
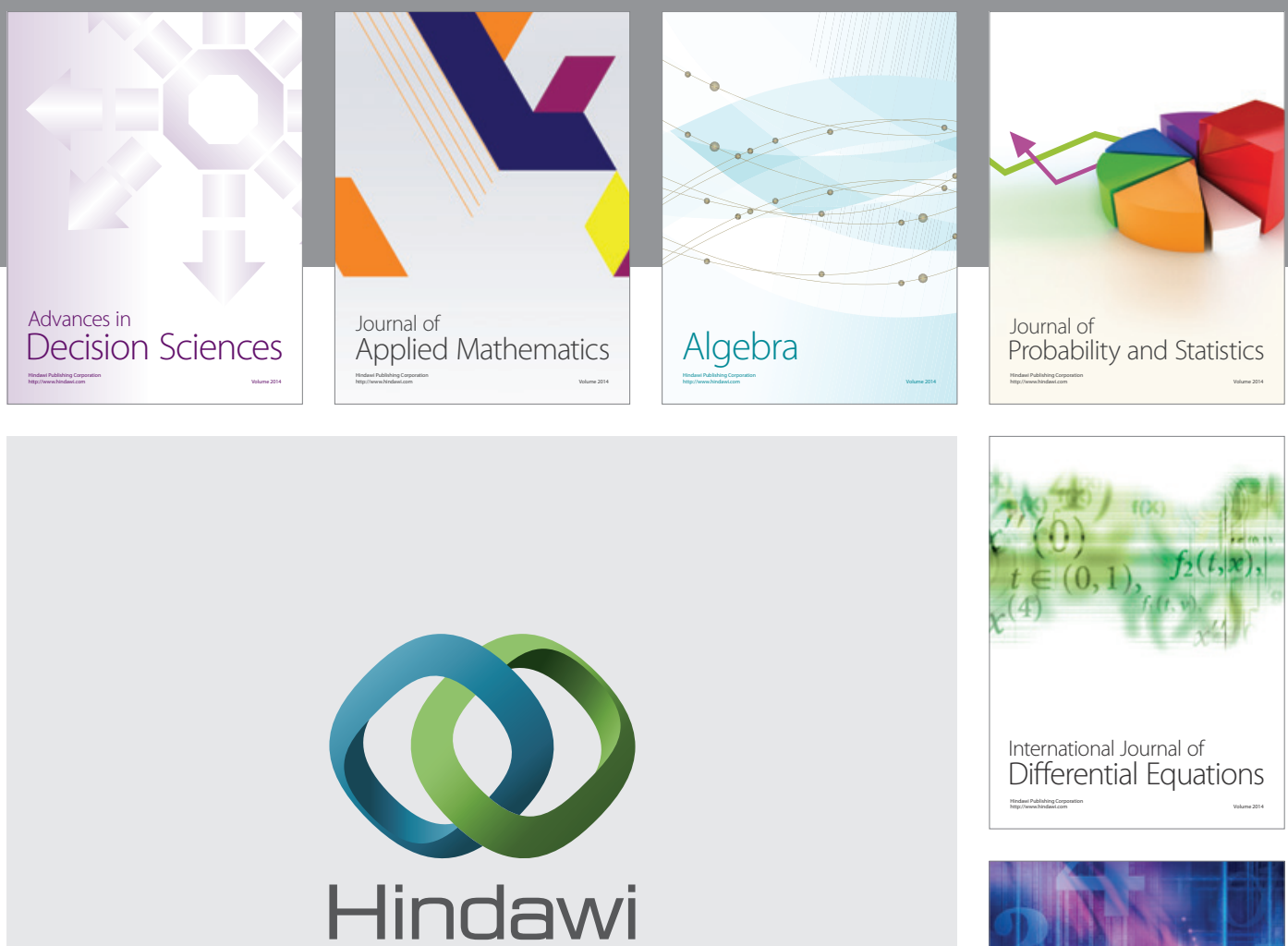

Submit your manuscripts at http://www.hindawi.com
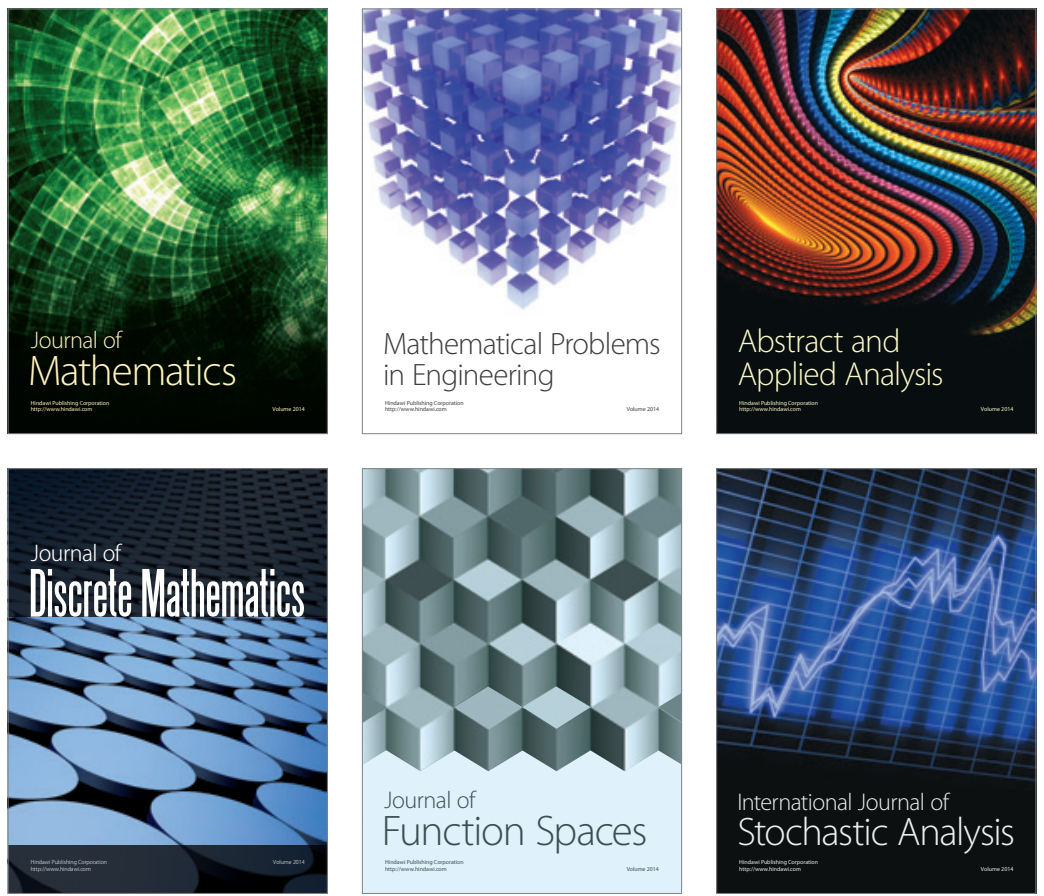

Journal of

Function Spaces

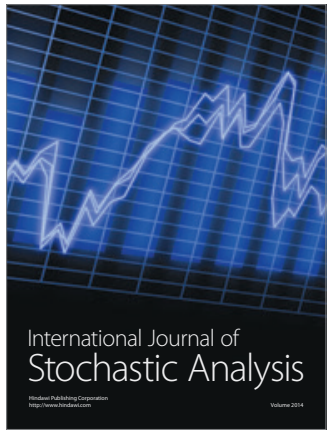

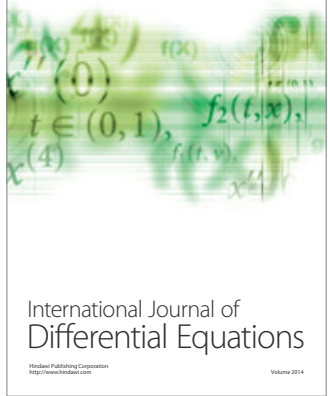
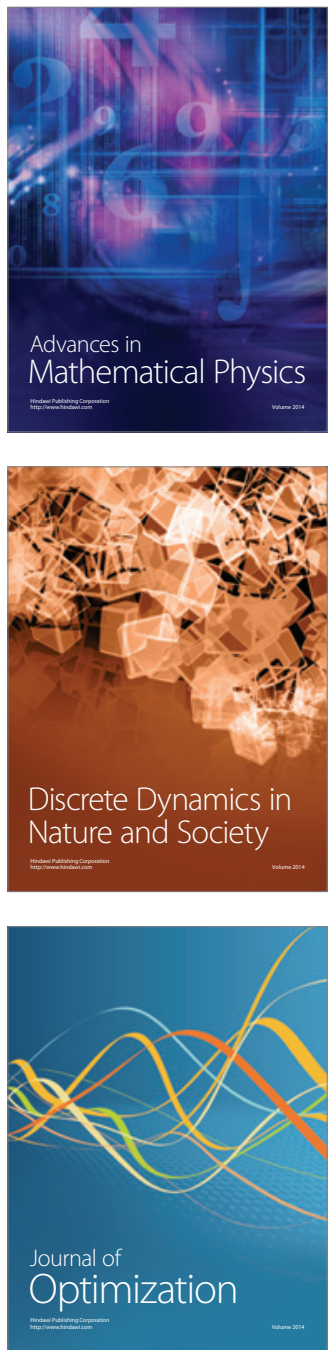